\title{
One-way FSI simulation of the phase and the geometric parameters of the model of compressor blades on the oscillating gas-dynamic processes
}

\author{
Butymova Liliia Nikolayevna and Modorskii Vladimir Yakovlevich \\ Federal State-Funded Education Institution of Higher Professional Education Perm National Research Polytechnic University, Center of \\ high-performance computing systems, Perm, Russia (29 Komsomolsky prospekt, Perm - GSP, Perm krai, Russia, 614990)
}

\begin{abstract}
The paper presents the results of numerical simulations to assess the impact of the phase shifts and the geometric design parameters on oscillations in inter-blade channel model compressor gas pumping unit. The blades are considered to be cantilevered plate. At low flow rates and at different angles of phase shift between oscillations of neighboring blades investigated gasdynamic forces acting on the structure. It is shown that the phase shift between the change in the corresponding gas-dynamic forces acting on the blades and vanes oscillations of depends on the phase shift between the oscillations of of the blades.
\end{abstract}

\section{Introduction}

Analysis of vibrating processes in compressors of gas pumping unit (GPU) shows that the complex vibrations occur comprising a "blade" and "rotor" and the other frequencies.

Investigation of mechanisms of formation of oscillations and the need to develop measures to prevent their extinction or requires computational tools to evaluate not only the frequency and amplitude of the oscillations, but also their phase. The latter is difficult, as it implies the study fast flowing multidimensional, multidisciplinary process, as in the cavity gas-dynamic, and in complex structures, taking into account the rotation.

In present article we study the effect of the phase model of compressor blades oscillations and geometric parameters of the structure on the amplitude, frequency and phase, acting on the blades of gas-dynamic forces. At the same time to facilitate the analysis does not take into account the vibration with the "rotary" frequencies for this selected low flow velocity along the blade, imitating the rotation at low speeds.

\section{Discussed problems}

The problem of occurrence and vibration reduction is closely associated with the problems of ensuring efficiency, noise reduction and meet the requirements of International Civil Aviation Organization (ICAO). There are a number of papers [1]-[13] associated with reduced vibration and noise, the problem is with the actual. This is due to the need to improve existing methods of designing and in-depth study of the mechanisms of occurrence of oscillatory processes.

\section{Physical model}

Physical statement of the problem should be formulated:

- processes are considered to be three-dimensional dynamic; there in the air, which is regarded as compressible as the working fluid; calculation scheme includes two fixed bodies that simulate two adjacent compressor blades; movable bodies are cantilevered; applied to the blades vibration load varying harmonically.

It is assumed that the blade can be independently the same oscillation amplitude and frequency but differing in phase. In this case the blades of gas-dynamic force is applied distributed. It is assumed that if the resultant gasdynamic forces of the in-phase component of the disturbing vibration, the vibrations may increase. If these oscillations occur with a phase shift, depending on its magnitude may both amplification and attenuation of vibrations. Compressor operation is modeled flow at a low speed oncoming. The phase shift between the oscillations of neighboring blades in the calculations was assumed to be fixed as follows: $0, \pi / 4, \pi / 2,3 \pi / 4, \pi$. Calculation scheme is shown in Fig. 1.

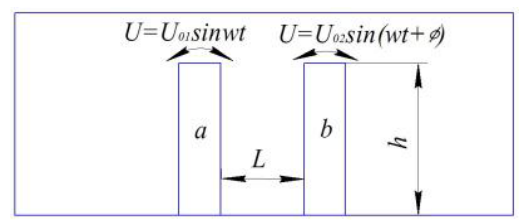

Figure 1. Calculation scheme

\section{Mathematical model}

The mathematical model includes the following relations: 
- law of conservation of mass

$$
\frac{\partial \rho}{\partial t}+\nabla(\rho \vec{V})=0
$$

- law of conservation of momentum

$$
\begin{aligned}
& \frac{\partial \rho \vec{V}}{\partial t}+\nabla(\rho \vec{V} \otimes \vec{V})= \\
& =-\nabla P+\nabla\left(\left(\mu+\mu_{t}\right)\left(\nabla \vec{V}+(\nabla \nabla)^{T}\right)\right)
\end{aligned}
$$

- law of energy conservation

$$
\frac{\partial(\rho H)}{\partial t}+\nabla(\rho \vec{V} H)=\frac{\partial P}{\partial t}+\nabla\left(\left(\frac{\lambda}{c_{p}}+\frac{\mu_{t}}{\mathrm{Pr}_{t}}\right) \nabla H\right)
$$

where $t$ - time; $\rho$ - density; $V$ - velocity; $P$ - pressure; $\mu$ - dynamic viscosity; $\mu_{t}$ - turbulent dynamic viscosity; $H$ - enthalpy; $c_{p}$ - heat capacity; $\lambda$ - coefficient of thermal conductivity; $\mathrm{Pr}_{t}$ - turbulent Prandtl number.

It uses well-known $k-\varepsilon$ model of turbulence. We introduce the following relations:

$$
\frac{\partial(\rho k)}{\partial t}+\nabla(\rho \nabla k)=\nabla\left(\left(\mu+\frac{\mu_{t}}{\sigma_{k}}\right) \nabla k\right)+\mu_{t} G-\rho \varepsilon,
$$

$\frac{\partial(\rho \varepsilon)}{\partial t}+\nabla(\rho \vec{V} \varepsilon)=$

$=\nabla\left(\left(\mu+\frac{\mu_{t}}{\sigma_{\varepsilon}}\right) \nabla \varepsilon\right)+C_{1} \frac{\varepsilon}{k} \mu_{t} G-C_{2} f_{1} \rho \frac{\varepsilon^{2}}{k}$

г д е $G=D_{i j} \frac{\partial V_{i}}{\partial x_{i}}, D_{i j}=S_{i j}-\frac{2}{3}\left(\nabla \cdot \vec{V}+\frac{\rho k}{\mu_{t}}\right) \delta_{i j}$, $S_{i j}=\frac{\partial V_{i}}{\partial x_{j}}+\frac{\partial V_{j}}{\partial x_{i}}, \sigma_{k}=1, \quad \sigma_{\varepsilon}=1,3, C_{1}=1,44$, $C_{2}=1,92, f_{1}=1, k$ - turbulent energy, $\varepsilon$ - turbulent energy dissipation rate, $G$ - determines the rate of generation of turbulent energy, $S_{i j}$ - double rate of deformation tensor.

The equation of turbulent viscosity is calculated using the formula of Kolmogorov-Prandtl:

$$
\mu_{t}=C_{\mu} \rho \frac{k^{2}}{\varepsilon},
$$

where $C_{\mu}=0,09-$ constant.

- equation of state

$$
P=\rho R T,
$$

where $R$ - gas constant.

Mathematical model closes initial and boundary conditions. The initial values of temperature and pressure as follows: $\mathrm{T}_{0}=20^{\circ} \mathrm{C}, \mathrm{P}_{0}=0.1 \mathrm{M} \Pi \mathrm{a}, \mathrm{V}_{0}=0,1 \mathrm{M} / \mathrm{C}$.

\section{Algorithm of numerical modelling}

Method of calculation was developed for the calculation of the gas-dynamic interblade channel, comprising the steps of:
1) Development calculated scheme, which is a tube of rectangular cross section, arranged in it two cantilevered blades.

2) Design of gas-dynamic cavity is the space between the blades, as well as the inlet and outlet pipes of the model.

3) Construction of the gas-dynamic calculation grid cavity is a box in one cell width.

4) It is necessary to assign to the lateral surface of the blade model compressor boundary condition «Wall», appoint perpendicular to the shaft axis of the plane on the surface bounding Gasdynamic cavity boundary condition «Symmetry», assign to the side surfaces of the blade condition «Wall», with a special option to Mesh Motion grid " Specified Displacement ».

5) Set properties of the gas flowing in the gas cavity. To do this, choose from a library of materials ANSYS CFX software package, you need a working body.

6) Define settings for solving the problem, convergence parameters and monitoring the progress of calculation in the software package ANSYS CFX, appointing Solver Control and Output Control option.

7) Identify the necessary resources to solve the problem. Running high-performance computing solutions to complex of Perm National Research Polytechnic University allows an assessment of the necessary cluster resources.

8) Processing gas-dynamic calculation of the results, the conclusion of necessary variables as fields fills, graphs, tables, vectors, streamlines.

\section{Computational examples and analysis}

If we consider the process of gas-dynamic flow between two adjacent blades of the rotor rim, it is possible in addition to the specification of "rotary" and "blade" of frequencies, to clarify the mechanism of occurrence of vibrations, namely in the part related to the spread of the gas-dynamic flow between the shoulder blades. As a first approximation, the analysis is reduced to the search for a model similar to the problem of motion of the gasdynamic waves in an open pipe or on the motion of the gas-dynamic waves in the open at one end of the tube. Significant factors in the analysis of this process is the interscapular distance and the height of the blades. In some cases, one factor dominates, in others - the other.

Tables 1, 2, 3 shows the relationship between the predetermined phase shift and structure oscillations obtained in the course of the numerical solution of the phase shift between gas-dynamic forces acting on the blades, and oscillations phase shift between the blade and the gas-dynamic force acting on it.

Table 1. Phase shifts in oscillations in the gas and construction at $\mathrm{L}=50 \mathrm{~mm}$ and a cantilevered

\begin{tabular}{|c|c|c|}
\hline \multirow{2}{*}{$\begin{array}{c}\text { C onstr } \\
\text { uction }\end{array}$} & Gas & Gasdynamics-construction \\
\cline { 3 - 3 } & dynamics & $\begin{array}{c}\text { The phase oscillations between } \\
\text { the blade and the gas-dynamic } \\
\text { force acting on it }\end{array}$ \\
\hline
\end{tabular}




\begin{tabular}{|c|c|c|c|c|c|}
\hline 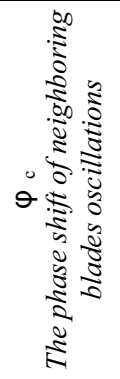 & 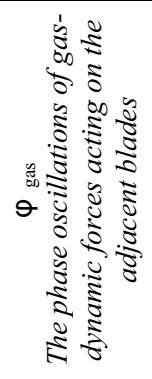 & $\stackrel{\frac{\pi}{0}}{\frac{\mathbb{J}}{0}}$ & 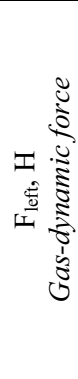 & 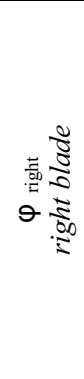 & 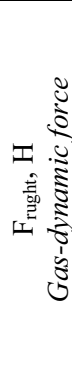 \\
\hline 1 & 2 & 3 & 4 & 5 & 6 \\
\hline 0 & 0 & $\begin{array}{l}0,- \\
\pi / 2\end{array}$ & 0,02 & $\begin{array}{l}0,- \\
\pi / 2\end{array}$ & 0,03 \\
\hline$\pi / 4$ & 0 & $\pi / 4$ & 0,6 & 0 & 0,69 \\
\hline$\pi / 2$ & $\pi$ & $-\pi / 2$ & $-0,8$ & $-\pi$ & 0,7 \\
\hline $3 \pi / 4$ & $-\pi / 2$ & $-\pi / 4$ & 0,6 & 0 & 0,63 \\
\hline & $\pi / 4$ & $-\pi$ & 0,01 & 0 & 0,01 \\
\hline
\end{tabular}

Table 2. Phase shifts in oscillations in the gas and construction at $\mathrm{L}=100 \mathrm{~mm}$ and a cantilevered

\begin{tabular}{|c|c|c|c|c|c|}
\hline \multirow{3}{*}{ 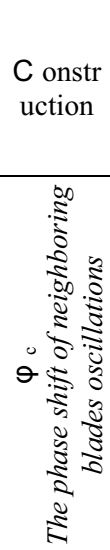 } & \multirow{3}{*}{ 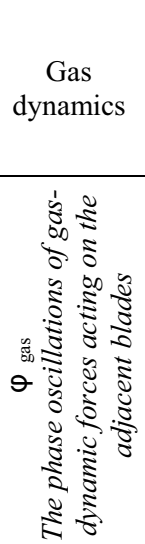 } & \multirow{2}{*}{\multicolumn{4}{|c|}{$\begin{array}{c}\text { Gasdynamics-construction } \\
\text { The phase oscillations between } \\
\text { the blade and the gas-dynamic } \\
\text { force acting on it }\end{array}$}} \\
\hline & & & & & \\
\hline & & $\theta \frac{\sqrt{2}}{\frac{\pi}{2}}$ & 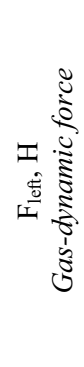 & 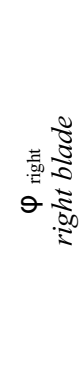 & 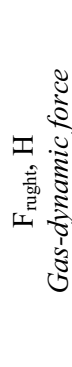 \\
\hline 1 & 2 & 3 & 4 & 5 & 6 \\
\hline 0 & 0 & 0 & 0,1 & 0 & 0,1 \\
\hline$\pi / 4$ & $-\pi / 2$ & $3 \pi / 4$ & 0 & $\pi / 2,0$ & 0,44 \\
\hline$\pi / 2$ & $\pi$ & $-\pi / 2$ & 1 & $-\pi$ & $-1,75$ \\
\hline $3 \pi / 4$ & $-\pi / 2$ & $-\pi / 2$ & 0,09 & 0 & 0,5 \\
\hline$\pi$ & $-\pi / 2$ & $-\pi / 4$ & 0,01 & 0 & 0,01 \\
\hline
\end{tabular}

Table 3. Phase shifts in oscillations in the gas and construction at $\mathrm{L}=139 \mathrm{~mm}$ and a cantilevered

\begin{tabular}{|c|c|c|c|c|c|}
\hline \multirow[b]{2}{*}{$\begin{array}{l}C \text { onstr } \\
\text { uction }\end{array}$} & \multirow[b]{2}{*}{$\begin{array}{c}\text { Gas } \\
\text { dynamics }\end{array}$} & \multicolumn{4}{|c|}{ Gasdynamics-construction } \\
\hline & & \multicolumn{4}{|c|}{$\begin{array}{c}\text { The phase oscillations } \\
\text { between the blade and the } \\
\text { gas-dynamic force acting on it }\end{array}$} \\
\hline 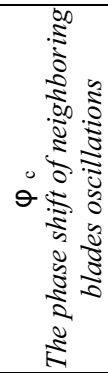 & 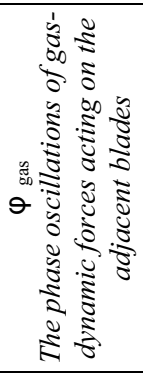 & $\theta \frac{\frac{\pi}{5}}{\frac{5}{5}}$ & 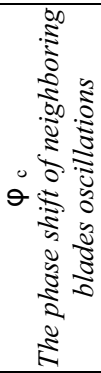 & 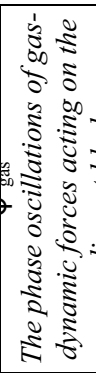 & $\theta \frac{\frac{\pi}{8}}{\frac{\pi}{0}}$ \\
\hline 1 & 2 & 3 & 4 & 5 & 6 \\
\hline 0 & 0 & 0 & 0,09 & 0 & 0,06 \\
\hline$\pi / 4$ & $-\pi / 2$ & $\begin{array}{l}\pi / \\
4\end{array}$ & 0,5 & $\pi / 4$ & 0,08 \\
\hline$\pi / 2$ & $\pi$ & $\pi /$ & -1 & $\pi / 2$ & 1.49 \\
\hline
\end{tabular}

\begin{tabular}{|c|c|c|c|c|c|}
\hline & & 2 & & & \\
\hline $3 \pi / 4$ & $-\pi / 2$ & $3 \pi$ & 0,58 & $3 \pi / 4$ & 0,18 \\
\hline$\pi$ & $-\pi / 2$ & $\pi$ & 0,01 & $\pi$ & 0,01 \\
\hline
\end{tabular}

Table 4. By the analysis of oscillatory processes

\begin{tabular}{|c|c|c|c|c|c|}
\hline $\mathrm{L}, \mathrm{m}$ & $h_{b}, m$ & $\begin{array}{c}\mathrm{a}, \\
\mathrm{m} / \mathrm{s}\end{array}$ & $\begin{array}{l}\mathrm{f}_{\mathrm{L}} \\
\mathrm{Hz}\end{array}$ & $\mathrm{f}_{\mathrm{h}}, \mathrm{Hz}$ & $f_{L} \backslash f_{h}$ \\
\hline 0,05 & \multirow{3}{*}{0,15} & \multirow{3}{*}{330} & 1320 & \multirow{3}{*}{2200} & 0,6 \\
\hline 0,1 & & & 6600 & & 3 \\
\hline 0,139 & & & 4700 & & 2,1 \\
\hline
\end{tabular}

Tables 1, 2, 3, F> 0 corresponds to a positive working gas dynamic forces, for $\mathrm{F}<0$ - negative. Analysis tables allowed to obtain dependence gas dynamic forces of inter-blade distance $(\mathrm{F}=\mathrm{f}(\mathrm{L}))$ for different shifts phases of neighboring blades $(\varphi)$. Example dependences obtained is shown in Fig. 2.
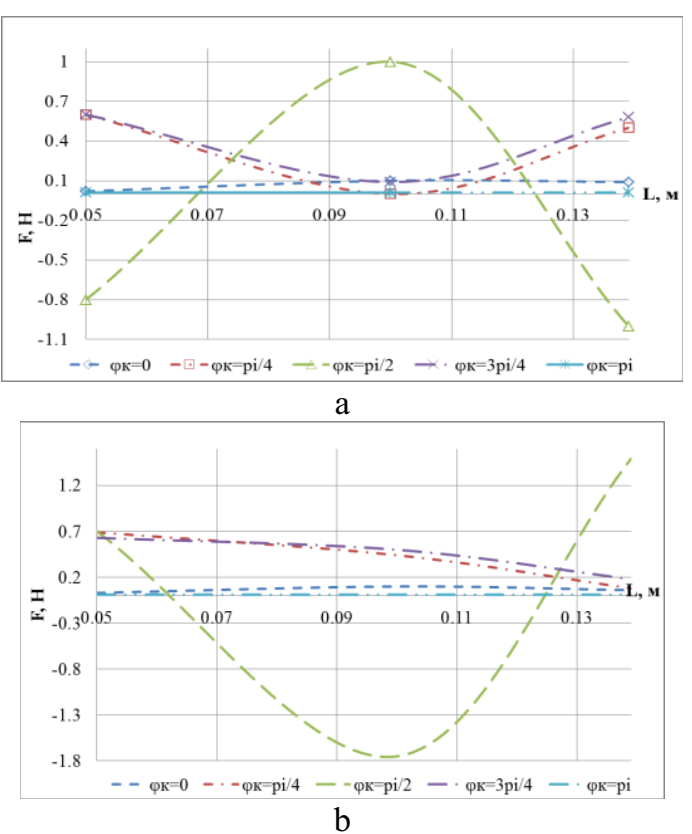

Figure 2. Dependence of gas dynamic forces from the inter-blade distance:

$$
\mathrm{a} \text { - left blade; } \mathrm{b} \text { - right blade }
$$

If inter-blade distance $\mathrm{L}$ is much greater than the height of the blade $\mathrm{h}_{л},\left(\mathrm{~L}>>\mathrm{h}_{л}\right)$ and $\varphi=0$, then:

- with oscillating movements of the blades in interblade space compression waves and expansion waves. They move toward each other and meet in the inter-blade space. Accordingly, real time, which is necessary for a travel is reduced by almost half $\left(L>>h_{л}\right)$. The natural frequency in this case is calculated as $\mathrm{f}=\mathrm{a} / \mathrm{L}$.

If the blade height $h_{л}$ much larger inter-blade distance dimensions L, namely $h_{л}>$ L:

- gas-dynamic flow is distributed in the inter-blade space as in a tube closed at one end. This changes the oscillation frequency of the gas-dynamic force, which is calculated according to the formula $\mathrm{f}=\mathrm{a} / 2 \mathrm{~L}$. In this case, the height of the blade determines the lower the natural frequency of the gas cavity between the shoulder blades $\left(\mathrm{h}_{л}>\mathrm{L}\right)$.

As a result of numerical experiment results were as follows. 
In Table $4 \mathrm{f}_{\mathrm{L}}$ - lower natural frequency inter-blade space in the tangential direction, $\mathrm{L}$ - the distance between blades, a - velocity of sound in the environment, $\mathrm{h}_{\mathrm{b}}-$ blade height, $\mathrm{f}_{\mathrm{h}}$ - lower natural frequency inter-blade space radially.

Table 4 shows that the inter-blade distance of $100 \mathrm{~mm}$, the resonance interaction occurs, since in this case there is a multiplicity of eigenfrequencies.

In deriving the dependency of the amplitude oscillations of the gas-dynamic forces interscapular distances for different phase shifts between the oscillations of the displacement of neighboring blades $(\varphi)$, the results were:

\section{If $\mathrm{L}=50 \mathrm{~mm}$ :}

- on the left blade, when the gas-dynamic work force is greater than zero $\left(A_{F}>0\right)$ there is a maximum amplitude in the gas-dynamic forces at $\varphi=3 \pi / 4$. When the gasdynamic work force is less than zero $\left(\mathrm{A}_{\mathrm{F}}<0\right)$, maximum gas dynamic force amplitude observed at $\varphi=\pi / 2$, minimum - at $\varphi=\pi$ (Fig. 2a);

- on the right blade, when the gas-dynamic work force is greater than zero $A_{F}>0$ maximum gas dynamic force amplitude observed at $\varphi=\pi / 2$, minimum - at $\varphi=\pi$ (Fig. 2b);

If $\mathrm{L}=100 \mathrm{~mm}$ :

- on the left blade, when the gas-dynamic work force is greater than zero $A_{F}>0$ maximum gas dynamic force amplitude observed at $\varphi=\pi / 2$, minimum - at $\varphi=\pi$ (Fig. 2a);

- on the right blade, when the gas-dynamic work force is greater than zero $A_{F}>0$ maximum gas dynamic force amplitude observed at $\varphi=3 \pi / 4$. When $A_{F}<0$ maximum gas dynamic force amplitude observed at $\varphi=\pi / 2$, minimum - at $\varphi=\pi$ (Fig. 2b);

If $\mathrm{L}=139 \mathrm{~mm}$ :

- on the left blade, when the gas-dynamic work force is greater than zero $A_{F}>0$ maximum gas dynamic force amplitude observed at $\varphi=3 \pi / 4$. When $A_{F}<0$ maximum gas dynamic force amplitude observed at $\varphi=\pi / 2$, minimum - at $\varphi=\pi$ (Fig. 2a);

- on the right blade, when the gas-dynamic work force is greater than zero $A_{F}>0$ maximum gas dynamic force amplitude observed at $\varphi=\pi / 2$, minimum - at $\varphi=\pi$ (Fig. 2b);

\section{Conclusions}

1) These studies make it possible to use them as a base for carrying out complicated calculations and analysis of the results, taking into account: real geometry, rotation at different speeds, two-way FSI - interaction.

2) The study results allow to specify the "blade" of the frequency and the factors influencing them, and thus to clarify the issues related to the resonant interaction in the system "gas - construction" during the vibrations

3) Installing bandage on the shoulder can afford to change the oscillation frequency, and tune out the resonant interaction in the system "gas construction."

\section{Acknowledgements}

The research work was supported by Russian Science Foundation under Grant No. 14-19-00877.

\section{References}

1. A. Shmakov, V. Modorskii, Modeling of oscillating processes in elements construction SRM, Scientific and Technical Volga region Bulletin, 6, 368-371 (2013).

2. E. Mekhonoshina, V. Modorskii, On a phase-shift of waves at the medium interface, Computer Optics, 39(3), 385 (2015).

3. G D Ujade and M B Bhambere, Review of Structural and Thermal Analysis of Gas Turbine Blade, International Journal of Mechanical Engineering and Robotics Research, 3 (2), 347-352, (2014).

4. A. Shmakov, V. Modorskii. Energy Conservation in Cooling Systems at Metallurgical Plants, Metallurgist, 59(9), 882-886 (2016)

5. A. Shmakov, V. Modorskii. Numerical Modeling of Gasdynamic Processes and Processes of Deformation in Compressor of Model Test Bench of the GasDistributing Unit, AMM, 799-800, 865-869 (2015)

6. M. Dimentberga, S. Kolesnikov, Vibrations in thechnics. Vibrations machines, structures and their elements (Moscow, Mashinostroyeniye, 1980)

7. V. Modorskii and other, The solution of engineering problem on the high-performance computer system of the Perm national research polytechnical university: monograph. (Perm national research polytechnical university, 2014)

8. Butymova L.N., Modorskii V.Ya., Petrov V.U., Numerical simulation of model compressor blade oscillation influence on gas-structure system, Scientific and Technical Volga region Bulletin, 6, 161-163 (2015).

9. Modorskii V.Ya., Sokolkin U.V., "Gasoelastic processes in power plants: monograph. (FIZMATLIT, 2007).

10. Balakrishnan, A. V., Aeroelastic flutter analysis in viscous flow: continuum models, 16 (3), 221 (2009)

11. Mekhonoshina E.V., Modorskii V.Ya., Petrov V.Yu, Numeric simulation of the interaction between subsonic. (Source of the Document CEUR Workshop Proceedings, Conference «Information Technology and Nanotechnology, (ITNT-2015), Mathematical Modeling) 211-218 (2015)

12. Ponik A. N., Modorskii V. Ya., Kozlova A. V., Sokolkin Yu. V., Numerical modeling of flow mixing and cooling processes in a perforated exhaust duct, Russian Aeronautics (Iz VUZ), 57(2), 181-186 (2014)

13. Modorskii V.Y., Petrov V.Y., Aeroelastic Deformation of a Solid-Propellant Rocket Engine During Supercharging, Russian Aeronautics (Iz VUZ), 54 (3), 272-275 (2011) 